\title{
On Parametric Response Characteristics of Beams With Multiple Transverse Cracks
}

\author{
U.K. Mishra and S.K. Sahu \\ Department of Civil Engineering, NIT, Rourkela-769008, India
}

\begin{abstract}
(Received 7 July 2012; accepted 14 February 2013)
This study deals with the parametric instability of a beam with multiple cracks. The variation of buckling load and dynamic stability regions, with respect to relative crack depth and position of cracks, are analysed using FEM. The loading on the beam is considered to be axial with a simple harmonic fluctuation with respect to time. The equation of motion represents a system of second order differential equations with periodic coefficients of the Mathieu-Hill type. The development of the regions of instability arises from Floquet's theory, and the periodic solution is obtained by Bolotin's approach using the finite element method. The stiffness matrix of the cracked beam element is obtained from the flexibility matrix of the intact beam, and the additional flexibility matrix due to the crack. The frequencies of vibration and buckling loads of the cracked cantilever beams reduce with the increase in crack depth and number of cracks. The onset of instability occurs earlier with the introduction of more cracks. The instability region for the crack location nearer to the fixed end occurs at a lower excitation frequency of the cracked beam. The vibration and instability results can be used as a technique for structural health monitoring or testing of structural integrity, performance, and safety.
\end{abstract}

\section{INTRODUCTION}

Many engineering structures may have structural defects, such as cracks, due to mechanical vibrations, environmental elements, corrosion, long term service, cyclic load, or etc. The presence of transverse cracks in beams may carry the potential risk of destruction and increases maintenance costs. This flexibility changes the dynamic behaviour of the structural member. Besides this, modern crack detection procedures, such as ultrasonic or X-rays, prove to be inoperative and unsuitable in many industrial situations due to detailed periodic inspections. Therefore, crack detection through vibration analysis is of tremendous technical importance. One of the most investigated approaches in detecting damage is the use of vibration data as a basis for structural health monitoring. Many considerations can contribute to the formation of a crack, and the potentially catastrophic consequences have made structural integrity testing an extremely active area of research. Hence, the dynamic characteristics of cracked beams are of considerable importance in many designs.

The effects of cracks upon the dynamic characteristics of beams are studied by many authors. Some details of earlier works on the topic has been reported in the review paper by Dimarogonas. ${ }^{1}$ Kisa et al. analysed the free vibration characteristics of cracked beams by the finite elements and component mode synthesis method. ${ }^{2}$ Yokoyama and Chen studied the vibration characteristics of a uniform Bernoulli-Euler beam with a single edge crack using a modified line-spring model. ${ }^{3}$ Saavedra and Cuitino presented the theoretical and experimental dynamic behaviour of a free-free beam with a transverse crack using the finite element method. ${ }^{4}$ Viola et al. proposed a procedure for identifying cracks in structures using model test data and the finite element method. ${ }^{5}$ Sinha and Friswell examined the vibration behaviour of a free-free beam with a breath- ing crack, simulated for a sinusoidal input force using the finite element method. ${ }^{6}$ Krawczuk et al. studied the dynamic behaviour of a cracked Timoshenko beam using the spectral element method. ${ }^{7}$ Zheng and Kessissoglu obtained the natural frequencies and mode shapes of a cracked beam using the finite element method. ${ }^{8}$ Loya et al. obtained the natural frequencies of vibrations of simply supported Timoshenko beams with cracks using a perturbation method. ${ }^{9}$

However, most of these studies involved the dynamic characteristics of beams with a single crack. Shifrin et al. proposed a new method for calculating the natural frequencies of a vibrating beam with an arbitrary finite number of transverse open cracks. ${ }^{10}$ Khiem et al. studied the free vibration characteristics of a beam with an arbitrary number of cracks, ${ }^{11}$ while Behera et al. used an analytical method for solving the frequency equation of an elastic beam with single and multiple cracks. ${ }^{12}$ Kisa and Gurel proposed a numerical model that combines the finite element and the component mode synthesis method for modal analysis of circular beams with non-propagating open crack. ${ }^{13}$ Kisa and Gurel further extended their study to the free vibration analysis of uniform and stepped circular beams with cracks using the same approach. ${ }^{14}$ Orhan studied free vibration analysis of a cracked beam in order to identify the crack in a cantilever beam by using the analytical method and finite element modelling in ANSYS. ${ }^{15}$ Viola et al. conducted a free vibration analysis of an axially loaded, cracked Timoshenko beam using the dynamic stiffness method. ${ }^{16}$

But the stability studies involving beams with cracks are scarce. Zheng and Fan investigated the vibration and stability of cracked hollow beams by applying Hamilton's principle and a modified Fourier series representing the vibration and buckling modes. ${ }^{17}$ Kishen and Kumar examined the behaviour of cracked beam-columns of different slenderness ratios subjected to axial and lateral loads using the finite ele- 